\title{
Microstructure evolution and mechanical properties of thin strip twin roll cast (TRC) Mg sheet
}

\author{
X. Yang, C.L. Mendis J.B. Patel and Z. Fan \\ BCAST, Brunel University London, Kingston Lane, Uxbridge UB8 3PH, United Kingdom
}

Keywords: Twin roll casting, AZ31, high shear melt conditioning

\begin{abstract}
:
TRC Mg alloys are hot rolled to further reduce the thickness of the TRC strip which results in a sheet material with a relatively strong basal texture which produces anisotropic tensile properties based on the test direction. The low force TRC concept developed at BCAST provides a pathway to produce thin strip that does not require further hot rolling to achieve the final desired thickness. The as TRC and homogenised AZ31 show a refined grain and very little centre line. The as TRC AZ31 has a tensile yield strength of $220 \mathrm{MPa}$ along the casting direction (CD) and $190 \mathrm{MPa}$ perpendicular to CD showing a very small yield anisotropy.
\end{abstract}

\section{Introduction}

The use of Mg alloys in automotive and personal electronic applications could be increased by the production of $\mathrm{Mg}$ sheet or strip in a more cost-effective manner that reduces or even eliminates the need for hot or cold rolling which is an inherent problem with hexagonal materials. [1, 2]. Twin roll casting (TRC) $[3,4]$ conventionally combines casting and hot rolling in a single step to produce a strip at or close to the final thickness. The solidification front of TRC strip has a complex dependence on alloy composition, feeding temperature of the melt, position of the tip of the tundish, speed of the rolls and the roll force on the solidifying strip [3]. As the melt leaves the tip region, solidification begins at the surface of the rolls and continues until the kissing point where the two strands of the solidified metal meet and the solidification of the strip is complete. This causes the solute rich liquid to segregate to the centre of the strip leading to the formation of central line segregation. This macro-segregation along the centre line decreases the ductility of as-cast strip, extends the homogenization time and increases the production cost [5, 6]. Therefore, majority of research in to twin roll casting consider dilute alloys which minimise the centre line segregation and only relatively small fraction of research on TRC Mg alloys concentrates on the development of solute rich $\mathrm{Mg}$ alloys that can be strengthened further through precipitation hardening after TRC.

\section{High shear melt conditioned TRC (HSMC-TRC)}

The high shear melt conditioning (HSMC) developed within BCAST when applied to twin roll casting reduced the macro-segregation observed along the centre line of the TRC strip by changing the solidification front which cause the grain structure to change from columnar grains observed in conventional TRC to more equi-axed grain structure. In conventional TRC process columnar grains form near the roll surface and grow towards the centre of the strip. During growth, the columnar grains 
reject solute atoms towards the centre of the strip decreasing the solidification temperature of the centre of the strip creating a deep sump [7]. This promotes the formation of brittle intermetallic particles along the centre line. In the case of HSMC-TRC process, enhanced heterogeneous nucleation results in the advance of equiaxed grains and a uniform solidification front from the roll surface to the centre of the strip and the solute rejection in to the liquid to create centre line segregation is insignificant. Figure 1 (a) and (b) show the conventional TRC strip without melt conditioning for AZ31 and AZ91 respectively. Both TRC strips contain significant amount of centre line segregation while AZ91 shows increased segregation. The HSMC-TRC strip did not show similar segregation at the centre line and retained a finer equi-axed grain structure from the surface of the strip to the centre, Figure 3 (b and d) respectively for AZ31 and AZ91. The HSMC-TRC strip shows significantly larger elongation to failure compared with the conventional TRC strip, even though the strips prepared through conventional TRC and MCTRC both have similar yield strengths [8]. Both conventional TRC and HSMC-TRC strips were also tested at elevated temperatures and HSMC-TRC strip shows significant improvement in elongation to failure compared with the conventional TRC strip [8]. Unlike the conventional TRC strip, where elongation to failure decreased with the increased test temperature MC-TRC strip showed continued increase in the elongation to failure.

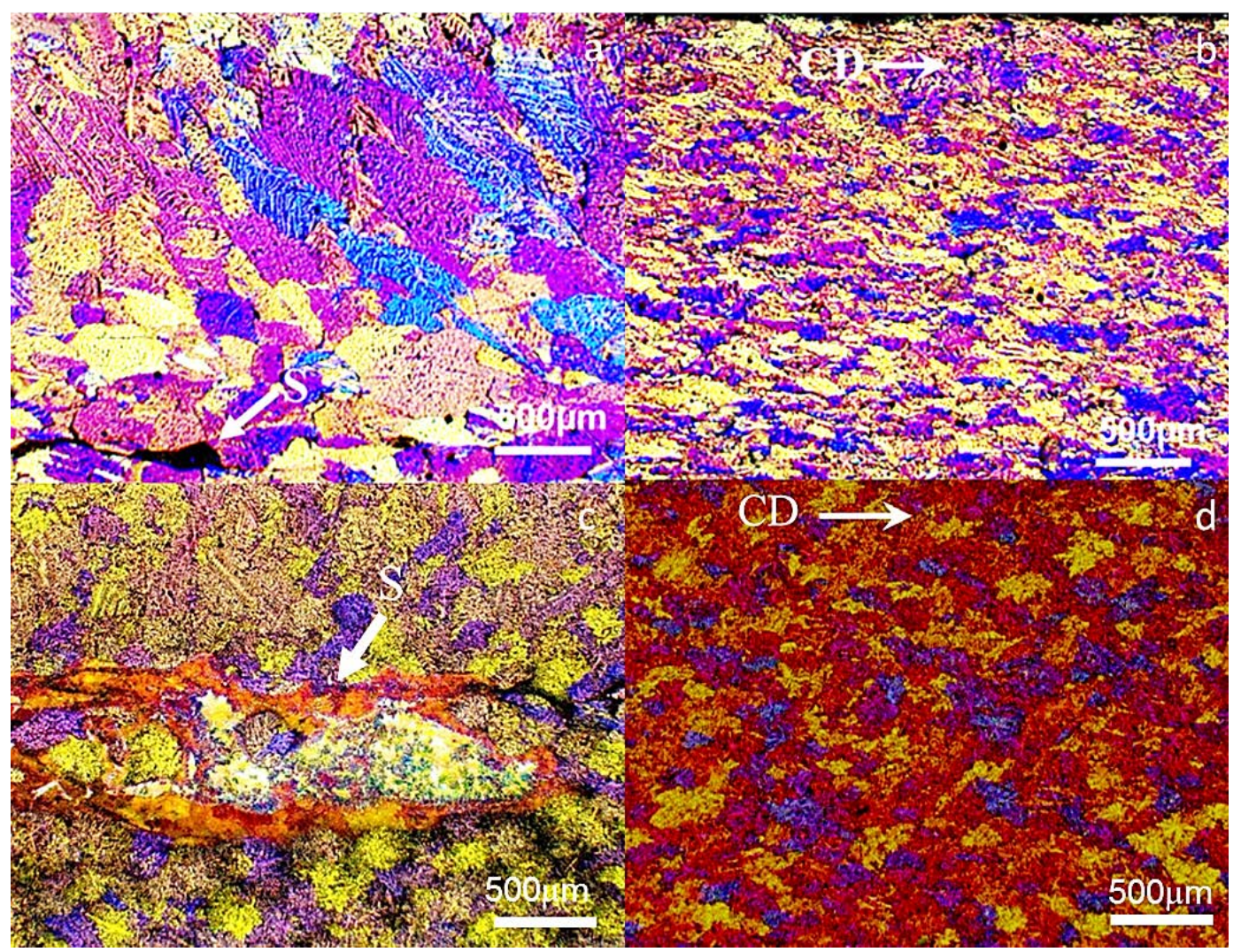

Figure 1. The microstructures of the as-cast TRC strips of (a, b) AZ31 and (c, d) AZ91 alloys prepared through (a .c) conventional TRC and (b, d) HSMC-TRC processes. Adopted from [9].

\section{Low force thin strip TRC}

The conventional TRC process produce a minimum strip thickness of approximately 3-10 mm, which needs to be subsequently hot rolled to produced $\mathrm{Mg}$ strip that may be used in personal electronics or automotive applications. Basal texture develops in majority of $\mathrm{Mg}$ alloys during the hot rolling reductions, reducing the formability of the TRC cast strip. Thus, removing the relatively randomised texture achieved during twin roll casting. The BCAST twin roll casting (BCAST-TRC) process is controlled by the solidification process and the low force on the rolls reduces the deformation of the 
solidified strip and retains the random texture distribution of the as-cast microstructure. The BCASTTRC provides just enough rolling force for strip thickness and surface quality control. The thin strip, low force TRC process in conjunction with melt conditioning provides a novel pathway for Mg sheet production [10]. The in-house twin roll caster has a small roll diameter and low separation force, and is capable of casting thin strip ( $<2 \mathrm{~mm}$ thickness). The setback between the tundish tip and kissing point of twin rolls is reduced by the small roll diameter as well as the reduced tip size. With smaller setback, the strip thickness is closer to the final gap due to reduced path from liquid metal to solid strip.

The as TRC strip produced via the BCAST-TRC show a refined microstructure and reduced segregation as compared with conventional TRC, Figure 2 (a) while combining the BCAST-TRC with high shear melt conditioning provides further refinement of the microstructure, Figure 2 (b). The reduction in the strip thickness changes the solidification front associated with the TRC reducing centreline segregation as the amount of heat to be extracted from the cross section of the strip is reduced. This is illustrated by the relatively `smaller central region containing coarse grains. The introduction of HSMC to the reduced thickness strip further modifies the solidification front to produce a strip consisting entirely of finer equi-axed grains. The thin strip prepared through the BCAST-TRC has a higher yield and tensile strengths along and perpendicular to the casting directions as compared with $5 \mathrm{~mm}$ TRC strip prepared under similar conditions, Figure 3. The yield strength of the AZ31 strip produced via BCAST-TRC has a minimum yield strength of $180 \mathrm{MPa}$ perpendicular to the casting direction and a maximum yield strength of 240MPa parallel to the casting direction. The $5 \mathrm{~mm}$ TRC strip however has a maximum yield strength of $200 \mathrm{MPa}$ parallel to the casting direction with the yield strength perpendicular to the casting direction reaching approximately $155 \mathrm{MPa}$. A yield strength of $240 \mathrm{MPa}$ is generally reported for the TRC and hot rolled AZ31 strip using conventional TRC processes [11] which is achieved through the BCAST-TRC in the as-cast condition.

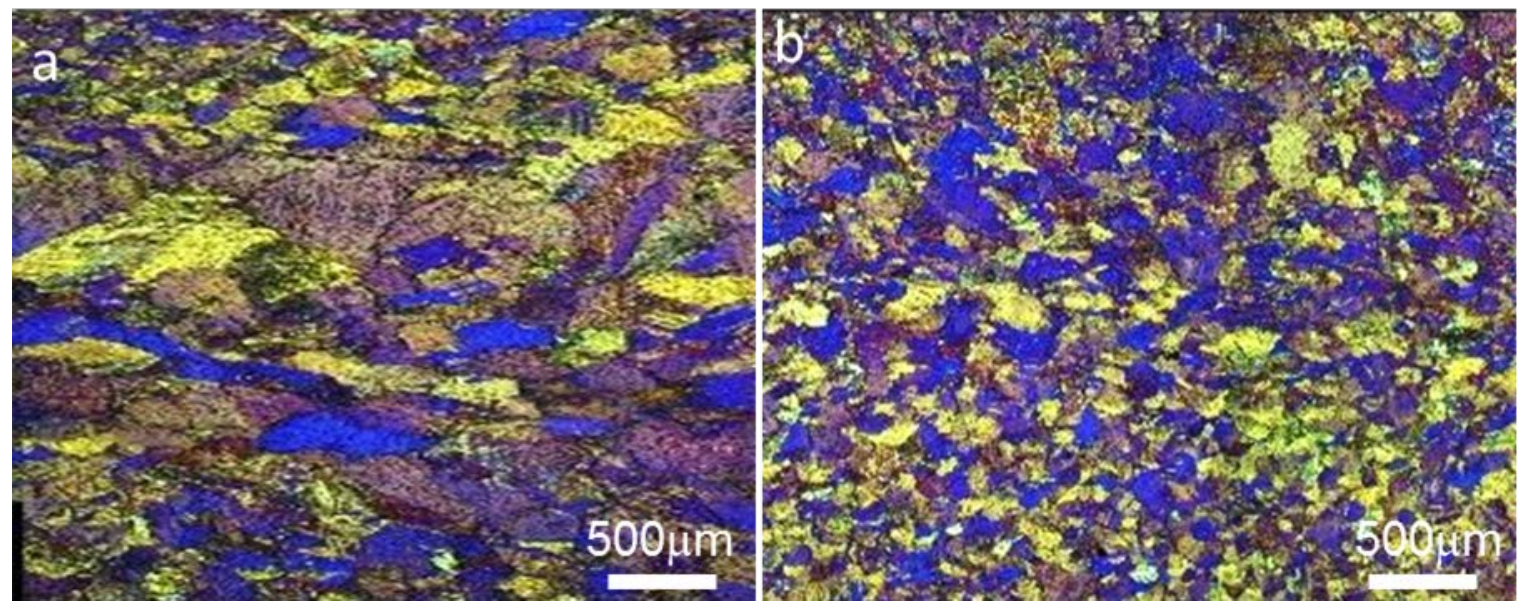

Figure 2. The microstructures of the thin strip AZ31 produced via BCAST-TRC process (a) without HSMC and (b) with HSMC. Adopted from [12].

\section{Conclusions}

The HSMC-TRC process with the application of melt conditioning through intensive melt shearing results in a uniform fine grain structure with reduced/eliminated centreline segregation. The thin strip produced using a BCAST low force twin roll caster has the potential to enable the development of high strength Mg alloy strip without a need for subsequent hot rolling with large thickness reductions. Thin gauge strip in combination with melt conditioning provides novel way to produce high quality TRC Mg strip providing an economical pathway to producing Mg sheet material. 


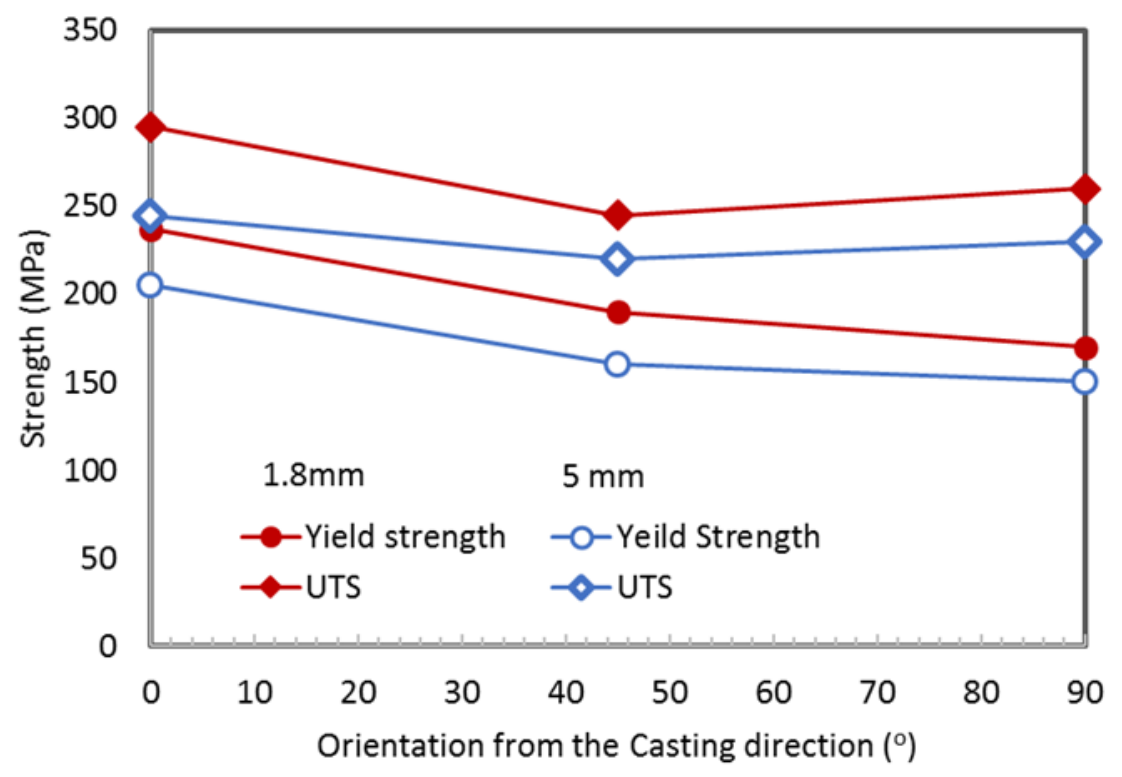

Figure 3. The yield and ultimate tensile strengths of as-TRC AZ31 alloy with a strip thickness of $5 \mathrm{~mm}$ and $1.8 \mathrm{~mm}$ based on the tensile test direction.

\section{References}

1. $\quad$ E. F. Emley; (1966) 'Principles of Magnesium Technology', Pergamon Press; London.

2. https://www1.eere.energy.gov/vehiclesandfuels/pdfs/alm_05/2j_herling.pdf. $\quad$ (accessed $14^{\text {th }}$ April 2017.

3. M. Ferry; (2006) 'Direct strip casting of metals and alloys', Woodhead publishing, London.

4. N. J. Kim, (2014) Critical Assessment 6: Magnesium sheet alloys: viable alternatives to steels? Mater. Sci. Tech., 30: 1925.

5. P. Bradbury, (1994) A mathematical model for the twin roll casting process D. Phil. Thesis, University of Oxford.

6. D. Liang, C. B. Cowley, (2004) The twin-roll strip casting of magnesium JOM, 56: 26.

7. S. Das et al. (2015) Effect of melt conditioning on heat treatment and mechanical properties of AZ31 alloy strips produced by twin roll casting Mater. Sci. Eng., A620: 223.

8. S. Das et al., (2013) Melt Conditioned Twin Roll Casting (MC-TRC) of Thin Mg-Alloy Strips for Direct Stamping of Mg Components Mater. Sci. Forum, 765: 170.

9. I. Bayandorian, (2010) Magnesium alloy strip produced by a Melt-conditioned twin roll casting process D. Phil. Thesis, Brunel University London.

10. Y. Huang et al. (2012) Microstructure control during twin roll casting of an AZ31 magnesium alloy, IOP Conference Series Mater. Sci. Eng., 27: 012065.

11. G. Kruz et al (2015) Rolling twin roll cast magnesium strips with varied temperature and degree of deformation Materials Today: Proceedings 2S: S39.

12. X. Yang (2016) Particle dispersion in Aluminium and Magnesium Alloys, D. Phil Thesis, Brunel University London. 